Products and Services In Practice is provided to readers using text and images from the manufacturer, supplier or distributor and does not imply endorsement by $B D J$ In Practice. Normal and prudent research should be exercised before purchase or use of any product mentioned.

Please send product and services news through to David Westgarth, BDJ In Practice via: David.Westgarth@bda.org

\section{A promise to customers}

At EndoCare we understand that root canal treatment can be daunting, especially for those who already suffer from dental anxiety or phobia.

That's why our whole team are dedicated to providing treatment that is as pain-free as possible. Using the latest techniques, root canal treatment need only be as uncomfortable as replacing a filling!

We also give patients the opportunity to listen to music while they are in the chair. This helps them relax and occupies their mind during treatment.

So why not refer your anxious patients to us? After treatment we will return them to your care with all the relevant aftercare notes and our suggested next steps.

It's the EndoCare promise. For further information call EndoCare on 0207224 0999 or visit www.endocare.co.uk.

\section{Confident clinical}

\section{decisions}

Make clinical observations easier with the CALCIVIS imaging system. With reliable, visual evidence of damaging demineralisation at an earlier stage than was previously possible, you can practice, better more efficient preventive dentistry.

This unique, chair-side visualisation device uses a bioluminescent photoprotein to detect microscopic free calcium ions as they are released from actively demineralising tooth enamel. It generates a glowing map of 'hot spots' to captivate

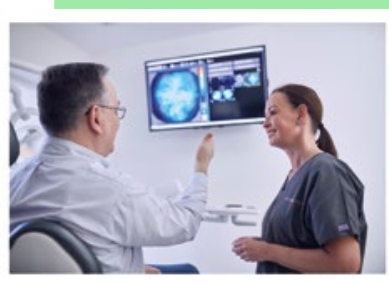
your patients and help them to understand their oral health status more effectively.

\section{Keep them coming back}

Designed with both patient and practitioner comfort in mind, the A-dec 500 supplied by Clark Dental features a double-articulating

access to the oral cavity.

It also combines a hydraulic lift with a virtual pivot system that is synchronised to the natural movement of the patient, eliminating the need for readjustment when the unit is raised or lowered.

With its multi-position armrests for streamlined entry and exit from the dental unit, the A-dec 500 is bound to keep patients coming back to your practice for a more comfortable treatment experience.

For more information, call Clark Dental on 01268733 146, email info@clarkdental. co.uk or visit www.clarkdental.co.uk.

\title{
The right choice
}

With so many electric toothbrushes available on the market, it can be difficult to recommend the right solution to your patients. Leading oral healthcare specialist, Curaprox, aims to make the choice simple with the Hydrosonic Pro (previously known as the Hydrosonic Ortho) toothbrush.

It is complete with three compact brush heads featuring ultra-fine CUREN filaments that facilitate fast, easy and thorough cleaning of the teeth and gums. This innovative device also boasts CURACURVE ergonomics that enable the user to manoeuvre the toothbrush into the most ideal position for effective removal of plaque - even in hard-to-reach areas.

With a powerful battery that ensures two weeks of cleaning for four minutes each day, the Hydrosonic Pro toothbrush offers outstanding mechanical and hydrodynamic performance.

For more information call 01480862084 , email info@curaprox.co.uk or visit www. curaprox.co.uk.

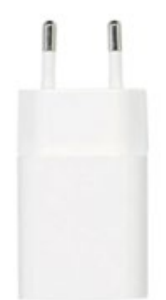

